\title{
Why is the mass function of NGC 6218 flat? ${ }^{\star}$
}

\author{
G. De Marchi ${ }^{1}$, L. Pulone ${ }^{2}$, and F. Paresce ${ }^{3}$
}

1 ESA, Space Science Department, Keplerlaan 1, 2200 AG Noordwijk, The Netherlands e-mail: gdemarchi@rssd.esa.int

2 INAF, Osservatorio Astronomico di Roma, Via di Frascati 33, 00040 Monte Porzio Catone, Italy e-mail: pulone@mporzio.astro.it

3 INAF, Viale del Parco Mellini 84, 00136 Roma, Italy e-mail: fparesce@inaf.it

Received 6 July 2005 / Accepted 28 November 2005

ABSTRACT

We have used the FORS-1 camera on the VLT to study the main sequence (MS) of the globular cluster NGC 6218 in the $V$ and $R$ bands. The observations cover an area of $3.4 \times 3.4$ around the cluster centre and probe the stellar population out to the cluster's half-mass radius $\left(r_{\mathrm{h}} \simeq 2\right.$ '2 $)$. The colour-magnitude diagram (CMD) that we derive in this way reveals a narrow and well defined MS extending down to the $5 \sigma$ detection limit at $V \simeq 25$, or about 6 magnitudes below the turn-off, corresponding to stars of $\sim 0.25 M_{\odot}$. The luminosity function (LF) obtained with these data shows a marked radial gradient, in that the ratio of lower- and higher-mass stars increases monotonically with radius. The mass function (MF) measured at the half-mass radius, and as such representative of the cluster's global properties, is surprisingly flat. Over the range 0.4-0.8 $M_{\odot}$, the number of stars per unit mass follows a power-law distribution of the type $\mathrm{d} N / \mathrm{d} m \propto m^{0}$, where, for comparison, Salpeter's IMF would be $\mathrm{d} N / \mathrm{d} m \propto m^{-2.35}$. We expect that such a flat MF does not represent the cluster's IMF but is the result of severe tidal stripping of the stars from the cluster due to its interaction with the Galaxy's gravitational field. Our results cannot be reconciled with the predictions of recent theoretical models that imply a relatively insignificant loss of stars from NGC 6218 as measured by its expected very long time to disruption. They are more consistent with the orbital parameters based on the Hipparcos reference system that imply a much higher degree of interaction of this cluster with the Galaxy than assumed by those models. Our results indicate that, if the orbit of a cluster is known, the slope of its MF could be useful in discriminating between the various models of the Galactic potential.

Key words. stars: Hertzsprung-Russell (HR) and C-M diagrams - stars: luminosity function, mass function Galaxy: globular clusters: general - globular clusters: individual: NGC 6218

\section{Introduction}

A satisfactory understanding of the properties of the initial mass function (IMF) of globular clusters (GCs) is a major objective of current astrophysical research in that GCs are the closest example of star formation at high redshift (Krauss \& Chaboyer 2003). The most reliable observations so far available that reach near the bottom of the stellar MS indicate that all halo clusters have a very similar present global MF (PGMF), which peaks at $\sim 0.35 M_{\odot}$ (Paresce \& De Marchi 2000; De Marchi et al. 2005). The internal dynamical relaxation process via stellar encounters is now reasonably well understood (Meylan \& Heggie 1997) and validated observationally (e.g. De Marchi et al. 2000; Albrow et al. 2002; Pasquali et al. 2004) and allows us to derive the global properties of the MF from a limited number of measurements within a cluster.

Nevertheless, any hope to infer useful information on the properties of the IMF from the observed present global MF

* Based on observations collected at the European Southern Observatory, Paranal, Chile, as part of programme 63.L-0423.
(PGMF) rests on our ability to roll back the effects that the tidal field of the Galaxy has exerted on the stellar population of the clusters (Paresce \& De Marchi 2000). Gravitational shocking due to repeated interactions with the bulge and disc of the Galaxy profoundly disrupt the original mass distribution by ejecting low mass stars from the core and by compressing the tidal boundary in phase space at each encounter. These phenomena, integrated over the orbit and time and eventually causing the disruption of the cluster, can substantially alter the shape of the MF thereby completely masking the properties of the IMF (Vesperini \& Heggie 1997).

Theoretical models describing the interaction of GCs with the Galactic tidal field have become progressively more detailed and, possibly, accurate in the past decade or so. This has been made possible by an in-depth analysis of the mechanisms responsible for cluster disruption (Aguilar et al. 1988; Gnedin \& Ostriker 1997) and by the availability of more accurate space motion parameters for the clusters (Dauphole et al. 1996; Odenkirchen et al. 1997). Models that make use of GC proper motion information (Dinescu et al. 1999; 
Baumgardt \& Makino 2003) should, in principle, provide a more reliable description of the clusters' past dynamical history than those purely based on radial velocity data (Gnedin \& Ostriker 1997). On the other hand, the models of Gnedin \& Ostriker (1997) explain rather convincingly, albeit in a statistical sense, why the GCs that we see today are not randomly distributed in parameter space but rather occupy regions of low probability of disruption.

The most useful indicator of the past dynamical history of GCs that models of this type produce is the time to disruption, $T_{\mathrm{d}}$, namely the time over which a cluster would be completely dissolved by tidal forces. Unfortunately, this parameter is not directly observable, thereby making it more difficult to assess the validity of the models. It is, therefore, necessary to relate $T_{\mathrm{d}}$ to other measurable parameters.

A rather obvious indication of a cluster's tidal disruption would seem to be the presence of an extended tidal tail (Leon et al. 2000; Odenkirchen et al. 2003). The unequivocal detection of tidal tails, however, is hard to achieve on the basis of photometric information alone (Baumgardt \& Kroupa 2005), which is often the only available data. A more robust, and potentially more powerful approach consists in looking at the properties of the MF of MS stars and at its variations within the cluster, with respect to that of other well behaved reference GCs. This has allowed us to identify, for the first time, the clear signature of tidal disruption in the cluster NGC 6712, in the form of a severe depletion of low-mass stars (De Marchi et al. 1999; Andreuzzi et al. 2001).

While our discovery has proved that GCs are indeed subject to the effects of tidal stripping, the widely different values of $T_{\mathrm{d}}$ predicted by various models for NGC 6712 have, at the same time, raised concerns as to the validity of the present physical understanding of the processes involved. In order to better understand the possible magnitude of the underlying discrepancy, we have studied the properties of the MF of another GC, NGC 6218, which, according to all three sets of presently available models (Gnedin \& Ostriker 1997; Dinescu et al. 1999; Baumgardt \& Makino 2003) should have experienced an insignificant or very mild interaction with the Galactic tidal field. We would expect that its PGMF should accurately reflect the IMF and our aim was to use NGC 6218 as a reference for NGC 6712 and other clusters. In this paper we report on the properties of its MF that shows that there may be something terribly wrong with these models or, more likely, with their assumptions.

The structure of the paper is as follows. The observations and their reduction are described in Sect. 2, whereas the results of the photometry are discussed in Sect. 3. Section 4 is devoted to the LF and MF of MS stars at various locations in the cluster. The dynamical structure of NGC 6218, as derived from these data, is presented in Sect. 5 and the overall implications for the understanding of the interaction between GCs and the potential field of the Galaxy are discussed in Sect. 6.

\section{Observations and data analysis}

The observations on which this paper is based were obtained in 1999 June with the FORS1 camera at ESO's Very Large
Table 1. Log of the observations.

\begin{tabular}{cccc}
\hline \hline Date UT & Filter & Exp. time (s) & Air mass \\
\hline 1999 Jun. 1 06:46:24 & $V$ & 180 & 1.232 \\
1999 Jun. 1 06:51:08 & $V$ & 180 & 1.246 \\
1999 Jun. 1 06:55:52 & $V$ & 180 & 1.261 \\
1999 Jun. 1 07:00:36 & $V$ & 180 & 1.277 \\
1999 Jun. 16 02:27:00 & $R$ & 180 & 1.163 \\
1999 Jun. 16 02:31:45 & $R$ & 180 & 1.154 \\
1999 Jun. 16 02:36:29 & $R$ & 180 & 1.146 \\
1999 Jun. 16 02:41:13 & $R$ & 180 & 1.138 \\
1999 Jun. 16 02:46:07 & $V$ & 180 & 1.131 \\
1999 Jun. 16 02:50:51 & $V$ & 180 & 1.124 \\
1999 Jun. 16 02:55:35 & $V$ & 180 & 1.118 \\
1999 Jun. 16 03:00:19 & $V$ & 180 & 1.112 \\
1999 Jun. 16 03:05:13 & $R$ & 180 & 1.107 \\
1999 Jun. 16 03:09:57 & $R$ & 180 & 1.103 \\
1999 Jun. 16 03:14:41 & $R$ & 180 & 1.099 \\
1999 Jun. 16 03:19:25 & $R$ & 180 & 1.095 \\
1999 Jun. 16 04:06:32 & $V$ & 180 & 1.086 \\
1999 Jun. 16 04:11:17 & $V$ & 180 & 1.087 \\
1999 Jun. 16 04:16:01 & $V$ & 180 & 1.089 \\
1999 Jun. 16 04:20:45 & $V$ & 180 & 1.092 \\
\hline
\end{tabular}

Telescope UT1, using the high resolution collimator with a field of view (FOV) of 3.4 on a side and a plate-scale of $0{ }^{\prime} 1$ per pixel. The log of the observations, carried out in service observing mode, is given in Table 1. Seeing conditions were excellent on both observing nights (1 June and 16 June), and the point spread function (PSF) had an average full width at half maximum of 0.6 . Observations were carried out in the Bessell $V$ and $R$ bands, with the exposure times listed in Table 1. The total equivalent exposure time corresponds to $2160 \mathrm{~s}$ in $V$ and $1440 \mathrm{~s}$ in $R$.

The telescope was pointed at the nominal centre of NGC 6218 at RA(J2000) $=16^{\mathrm{h}} 47^{\mathrm{m}} 14^{\mathrm{s}} 5$ and $\operatorname{Dec}(\mathrm{J} 2000)=$ $-1^{\circ} 56^{\prime} 52^{\prime \prime}$, 1 , corresponding to Galactic coordinates $l=15.7$ and $b=26.3$. Thanks to the relatively wide FOV of FORS-1, our observations reach out to the cluster's half-mass radius $\left(r_{\mathrm{h}}=2\right.$ '.16; Harris 1996) and comfortably contain the region within twice the core radius $\left(r_{\mathrm{c}}=0\right.$ '.72; Harris 1996).

The raw data were processed using the standard VLT pipeline calibration and photometry was later performed using the DAOPHOTII/ALLFRAME package (Stetson 1987, 1994). In particular, we first performed a photometric reduction using the daophot, and allstar tasks in order to build a preliminary list of stars on each single exposure. These lists were used to compute the coordinate transformation between each individual frame and a reference image. All the exposures, regardless of their wavelength band, could thus be matched and combined to obtain a median image, free from cosmic ray hits and with the highest signal-to-noise ratio for the final star finding procedure. The latter was performed in two steps, first by applying the routines daofind and allstar on the 


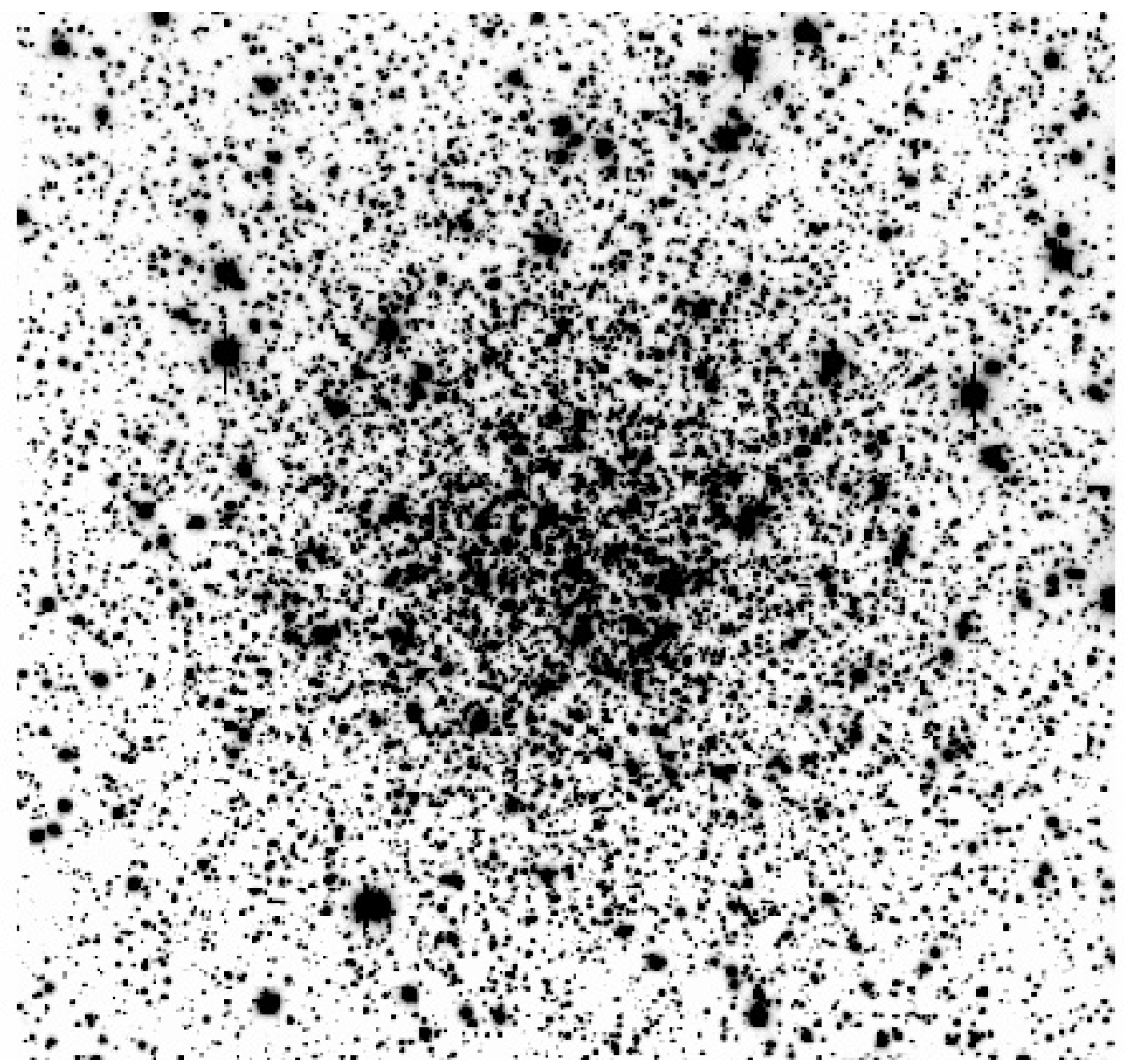

Fig. 1. Negative image of the cluster NGC 6218 of $180 \mathrm{sec}$ duration in the Bessell $V$ band. North is up and East to the left. The field spans 3.4 on a side.

stacked, deep multi-filter image, by setting the detection threshold at $5 \sigma$ above the local background level. We then analysed the PSF-subtracted image to recover objects missed in the first step. The final star list was then used as input to allframe for simultaneous PSF-fitting photometry of all of the objects in the individual frames. In order to take proper account of the geometric distortion of the optics, we have devised an automated procedure to define the best PSF candidate objects in each region of our target field.

The overall procedure above detected about 16000 bona fide objects in NGC 6218. Their instrumental magnitudes were finally transformed to the standard Johnson system by using the photometric standard field PG 2213, observed with the same instrumental set-up during our runs.

Our $5 \sigma$ detection limits correspond to magnitudes $V \simeq 25$ and $R \simeq 24$, where the photometric error reaches $\sim 0.1 \mathrm{mag}$.

\section{The colour-magnitude diagram}

The $V, V-R C M D$ of the complete set of 16015 objects that we have detected is shown in Fig. 2. At magnitudes brighter than $V \simeq 18$ the CMD clearly shows the sequences of stars evolved off the MS (sub-giant, red-giant and horizontal branches), as well as a conspicuous population of blue straggler stars. All these objects are discussed in detail in a forthcoming paper (Sabbi et al. 2006). Here we concentrate on the cluster MS, which is narrow and well defined from the turn-off at $V=18.8$, where the photometric error is small $\left(\sigma_{V}=0.01, \sigma_{R}=0.01\right)$, through to $V=24$, where the error on the magnitude grows to $\sigma_{V}=0.04$ and $\sigma_{R}=0.04$. At $V \simeq 25$, where our $5 \sigma$ detection limit for the star-finding algorithm falls, the increasing photometric error makes it difficult to distinguish the MS from possible contaminating field stars. The photometric errors and detection limit given here represent an average value over the whole image, but given the considerable density gradient of our target field the detection limit is brighter in the innermost regions and results in a lower photometric completeness there, as we explain in Sect. 4.

Figure 3 shows, traced over the same CMD of Fig. 2, the MS ridge line (solid curve) obtained by applying a sigmaclipping method to the colour of the stars along the MS, as explained in Sect. 4. The dashed line in the same figure corresponds to the theoretical isochrone computed by Baraffe et al. (1997) for a $10 \mathrm{Gyr}$ old cluster with metallicity $[\mathrm{M} / \mathrm{H}]=-1$ 


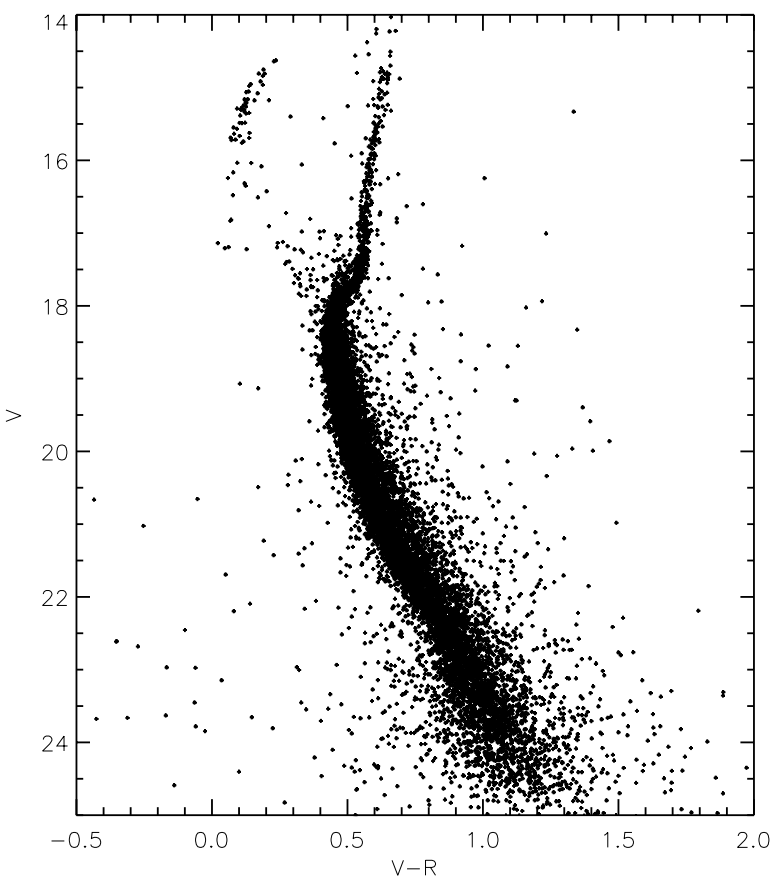

Fig. 2. Colour-magnitude diagram of an area of 3.4 $\times 3.4$ around the centre of NGC 6218 .

and helium content $Y=0.25$, as is appropriate for NGC 6218 . The excellent fit shown in Fig. 3 corresponds to a distance modulus $(m-M)_{V}=14.23$ and colour excess $E(B-V)=$ 0.18 , which compare favourably with the recently obtained $(m-M)_{V}=14.11$ and colour excess $E(B-V)=0.19$ of Hargis $\&$ Sandquist (2004). The difference in the distance modulus is small and fully consistent with the uncertainty on our absolute photometric calibration, and it would vanish if we were to take the distance modulus of Sato et al. (1989), namely $(m-M)_{V}=14.25$. We note here that the small discrepancy between the observed MS ridge line and that predicted by theoretical models at the lowest mass end (see Fig. 2) is a well known limitation of the theory and most probably stems from the lack of a proper treatment of the TiO molecular opacity, as extensively discussed in Baraffe et al. (1998; see also Chabrier 2001). The discrepancy becomes progressively smaller at longer wavelengths (Pulone et al. 2003, 1998). In any case, as we explain in the following section, this small uncertainty does not affect our conclusions on the mass distribution of MS stars, because over the mass range covered by our observations $\left(\sim 0.3-0.8 M_{\odot}\right)$ an uncertainty of $0.1 \mathrm{mag}$ in $V$ translates to an uncertainty of $\sim 0.01 M_{\odot}$ on the mass.

In order to study possible changes of the stellar population with position in the cluster, we have defined four distinct concentric regions over the area of our images. The innermost three are annuli $30^{\prime \prime}$ wide, while the fourth region includes all objects farther than $90^{\prime \prime}$ from the cluster centre through to the edge of the frame. For simplicity, hereafter we refer to these regions as "centre" and "rings" 1 through 3 . They contain, respectively, 1664, 4234, 5469 and 4648 objects. If instead of fixing the width of the rings we had kept constant their area or the number of stars in them, the radial extent of the annuli would have obviously changed. On the other hand, we have verified

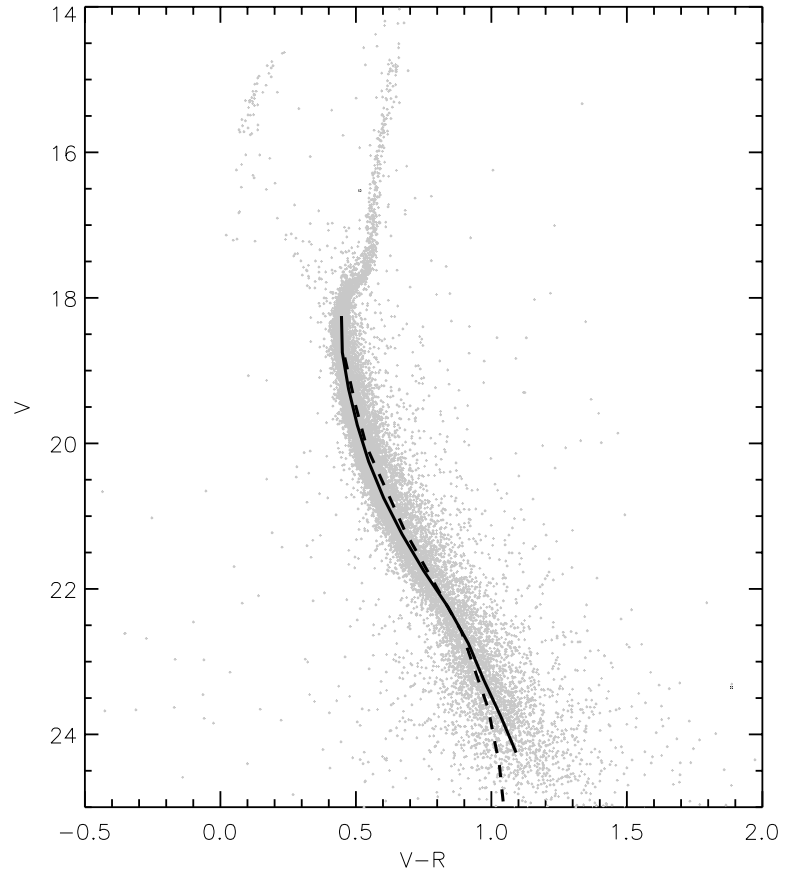

Fig. 3. Main sequence ridge line of NGC 6218 (solid curve) compared with the theoretical isochrones of Baraffe et al. (1997; dashed line) for an age of $10 \mathrm{Gyr}$ and metallicity $[\mathrm{M} / \mathrm{H}]=-1.0$. The best fit of the isochrones to the observed MS requires a distance modulus $(m-M)_{V}=$ 14.23 and a colour excess $E(B-V)=0.18$, consistent with the most recent estimates of Hargis \& Sandquist (2004).

that the progressive variation of the CMD and LF with radius is largely insensitive to the exact size of the rings.

The CMDs of the individual regions are shown in the four panels of Fig. 4. The most notable differences among the four panels is the marked central concentration of the blue stragglers, which are totally absent in the third ring, and the remarkable lack of bright red giants in the cluster centre, where the red giant branch stops one to two magnitudes fainter than outside. These issues are discussed elsewhere (Sabbi et al. 2006).

Also noteworthy in Fig. 4 is the rapid fading of the MS in the cluster centre well before the onset of significant photometric incompleteness. For instance, at $V \simeq 22$ the completeness is still above $50 \%$ (see below) and the photometric error small $\left(\sigma_{V}=0.03, \sigma_{R}=0.03\right)$, but the MS is already so sparse in the CMD that it cannot be distinguished from field stars. This finding is already a clear indication of the mass segregation that accompanies the normal dynamical relaxation process of GCs. Due to the equipartition of energy among stars, less massive objects tend to increase their kinetic energy and move onto larger orbits that force them outwards, away from the cluster centre, for most of their orbital period.

\section{The luminosity and mass function}

In order to study this effect in a more quantitative way, we decided to derive the LF of MS stars at various locations inside the cluster. In particular, one has to pay special attention to photometric incompleteness, which acts differentially on the LF and affects more prominently the denser central regions and 


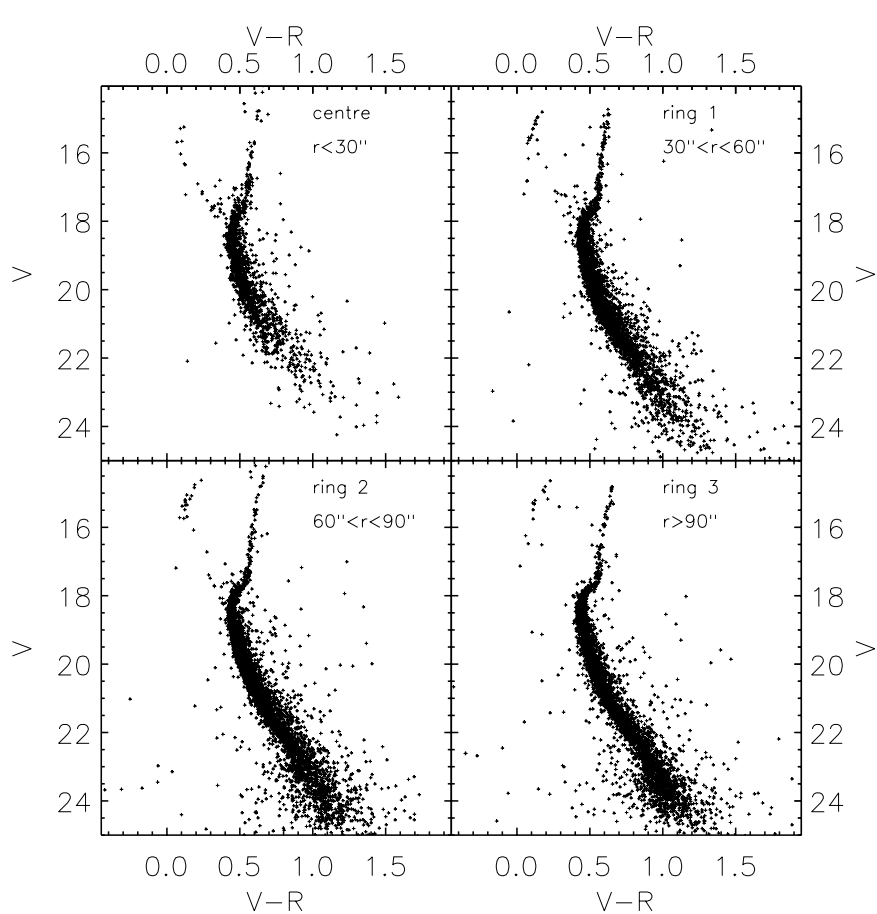

Fig. 4. Individual CMDs of the four regions in which we have divided our NGC 6218 field.

less or marginally the outer rings. Incompleteness is due to crowding and to saturated stars, whose bright halo can mask possible faint objects in their vicinity, both more likely to affect substantially the central regions (see Fig. 1). If ignored or corrected for in a uniform way across the whole image, by applying the same correction to all regions, these effects would bias the final results and mimic the presence of a high degree of mass segregation. For this reason, we conducted a series of artificial star experiments for the four individual regions (core and three rings), using the most appropriate local PSF for each.

The artificial star tests were run on the combined images, in both bands. For each 0.5 mag bin we carried out 10 trials by adding a fraction of $10 \%$ of the total number of objects (see Sect. 2). These trials were followed by running the tasks daophot.daofind, daophot.allstar and daophot.allframe, with the same parameters used in the reduction of the scientific images so that we could assess the fraction of objects recovered by the procedure and the associated photometric errors. The resulting photometric completeness is given in Table 2 for each region as a function of the $V$ magnitude, along with the associated $1 \sigma$ uncertainty.

In order to derive the LF from the CMDs of Fig. 4, one needs to separate true MS stars from field objects. The latter are however not significant at the Galactic latitude of NGC6218 ( $b=26.3)$, as Fig. 2 already shows. The Galaxy model of Ratnatunga \& Bahcall (1985) predicts about 17 field stars per $\operatorname{arcmin}^{2}$ towards the direction of NGC 6218 down to magnitude $V=25$, with about half of them in the range $23<V<25$. This would correspond to $\sim 200$ contaminating field stars in our FOV down to $V=25$ of which $\sim 100$ brighter than $V=23$. The CMD of Fig. 2 has of order 2000 stars in the range $V \geq 23$, before any correction
Table 2. Photometric completeness $f$ and the associated $1 \sigma$ uncertainty, both in percent, in the four regions in which we have divided our NGC 6218 field.

\begin{tabular}{ccccccccc}
\hline \hline \multicolumn{1}{c}{ Centre } & \multicolumn{3}{c}{ Ring 1} & \multicolumn{3}{c}{ Ring 2} & \multicolumn{3}{c}{ Ring 3 } \\
$V$ & $f$ & $\sigma$ & $f$ & $\sigma$ & $f$ & $\sigma$ & $f$ & $\sigma$ \\
\hline 18.25 & 91 & 2 & 94 & 1 & 95 & 2 & 96 & 1 \\
18.75 & 86 & 3 & 93 & 2 & 94 & 2 & 96 & 1 \\
19.25 & 79 & 6 & 92 & 4 & 93 & 2 & 95 & 1 \\
19.75 & 76 & 5 & 90 & 2 & 91 & 1 & 94 & 1 \\
20.25 & 75 & 4 & 86 & 4 & 89 & 1 & 92 & 1 \\
20.75 & 67 & 7 & 82 & 2 & 86 & 2 & 90 & 1 \\
21.25 & 59 & 9 & 77 & 2 & 83 & 2 & 88 & 2 \\
21.75 & 47 & 6 & 70 & 2 & 80 & 2 & 84 & 2 \\
22.25 & 34 & 5 & 62 & 5 & 74 & 2 & 80 & 2 \\
22.75 & 22 & 4 & 48 & 4 & 63 & 2 & 72 & 1 \\
23.25 & 13 & 4 & 31 & 3 & 49 & 3 & 59 & 2 \\
23.75 & 6 & 3 & 18 & 3 & 33 & 2 & 42 & 2 \\
24.25 & 2 & 1 & 7 & 3 & 19 & 4 & 23 & 2 \\
\hline
\end{tabular}

for incompleteness, and there are approximately 14000 stars brighter than that magnitude. Therefore, it would seem perfectly unnecessary to take field star contamination into account.

On the other hand, since field star contamination affects more prominently the lower end of the MS, which is most relevant to our investigation, we decided to remove it at all magnitudes. To this aim, we made use of the colour information in the CMD and applied the $\sigma$ clipping criterion described by De Marchi \& Paresce (1995) to identify the possible outliers. In practice, from the CMDs of Fig. 4 we measured the LF of MS stars by counting the objects in each 0.5 mag bin and within \pm 5 times the colour standard deviation around the MS ridge line and rejected the rest as field objects. This procedure identifies about 350 field stars in the magnitude range $18<$ $V<23$ and about 50 at fainter magnitudes. When photometric incompleteness is taken into account, the resulting number of contaminating stars is approximately a factor of four higher than that predicted by the model of Bahcall \& Soneira (1985). It is, nevertheless, very small ( $2 \%)$ when compared to the rest of bona fide MS stars and, in each magnitude bin, it is smaller than the statistical uncertainty associated with the counting process. In the following, we will consider only bona fide MS stars as defined in this way but, as will become clear in the rest of the paper, our conclusions would remain unchanged had we decided to ignore field star contamination and treat all stars as cluster members.

The average MS fiducial points are drawn as a solid line in Fig. 3 and are listed in Table 3 together with the colour width of the MS $\left(\sigma_{V-R}\right)$. Table 4 gives the four LFs, before and after correction for photometric incompleteness, and the corresponding rms errors coming from the Poisson statistics of the counting process (only for the LF corrected for incompleteness). All values have been rounded off to the nearest integer. The data are also shown graphically in Fig. 5, where the squares give the 
Table 3. Average main sequence fiducial points and colour width.

\begin{tabular}{ccc}
\hline \hline$V$ & $V-R$ & $\sigma_{V-R}$ \\
\hline 18.25 & 0.447 & 0.016 \\
18.75 & 0.450 & 0.017 \\
19.25 & 0.473 & 0.021 \\
19.75 & 0.505 & 0.027 \\
20.25 & 0.547 & 0.029 \\
20.75 & 0.603 & 0.035 \\
21.25 & 0.670 & 0.045 \\
21.75 & 0.750 & 0.050 \\
22.25 & 0.840 & 0.058 \\
22.75 & 0.915 & 0.064 \\
23.25 & 0.971 & 0.078 \\
23.75 & 1.034 & 0.094 \\
24.25 & 1.091 & 0.131 \\
24.75 & 1.173 & 0.150 \\
\hline
\end{tabular}

LF of the four regions, with the centre at the bottom and the outermost ring at the top. As Fig. 5 shows, all four LFs are remarkably flat, with the two central rings displaying a sensible drop in the number counts at low masses for $M_{V}>6.5$ (see upper axis). This result is very robust, because only data-points with an associated photometric completeness in excess of $50 \%$ are shown in Fig. 5.

A flat or dropping LF (i.e. one that drops with increasing magnitude) is not unusual in the core of GCs, where the effects of mass segregation are strongest. Such is, for instance, the case of 47 Tuc (Paresce et al. 1995), NGC 6397 (King et al. 1995) and NGC 7078 (De Marchi \& Paresce 1996; Pasquali et al. 2004). What is most unusual, however, is a flat LF near the cluster's half-mass radius, where a sample of 12 GCs studied by us (Paresce \& De Marchi 2000) has revealed a remarkably consistent behaviour with a LF increasing monotonically from the TO luminosity to $M_{V} \simeq 10$. For NGC 6218 this is clearly not the case, since the top LF in Fig. 5 samples the stellar population from a radius of $90^{\prime \prime}$ through to $155^{\prime \prime}$, with an equivalent radius of $115^{\prime \prime}$, comfortably reaching the nominal cluster's half-mass radius at $r_{\mathrm{h}}=2$ '.16 (Harris 1996). While near the half-mass radius of NGC 6397 the number of stars per unit magnitude grows by a factor of about four from the TO luminosity to $M_{V} \simeq 10$ (King et al. 1998), this number remains practically constant for NGC 6218. So far, the only GCs known to have a dropping or flat LF near the half-mass radius are NGC 6712 (De Marchi et al. 1999) and Pal 5 (Koch et al. 2004) and both are expected to have undergone a strong dynamical interaction with the Galactic tidal field, at variance with the expectations for NGC 6218.

The LFs shown in Fig. 5 can in principle be converted into MFs, since we have shown in Fig. 3 that the cluster MS is well fitted, within the theoretical and observational errors, by the available theoretical models of low-mass stars (Baraffe et al. 1997). We can, thus, reasonably expect these models to provide a reliable relationship between luminosity and mass. On the other hand, in order to keep

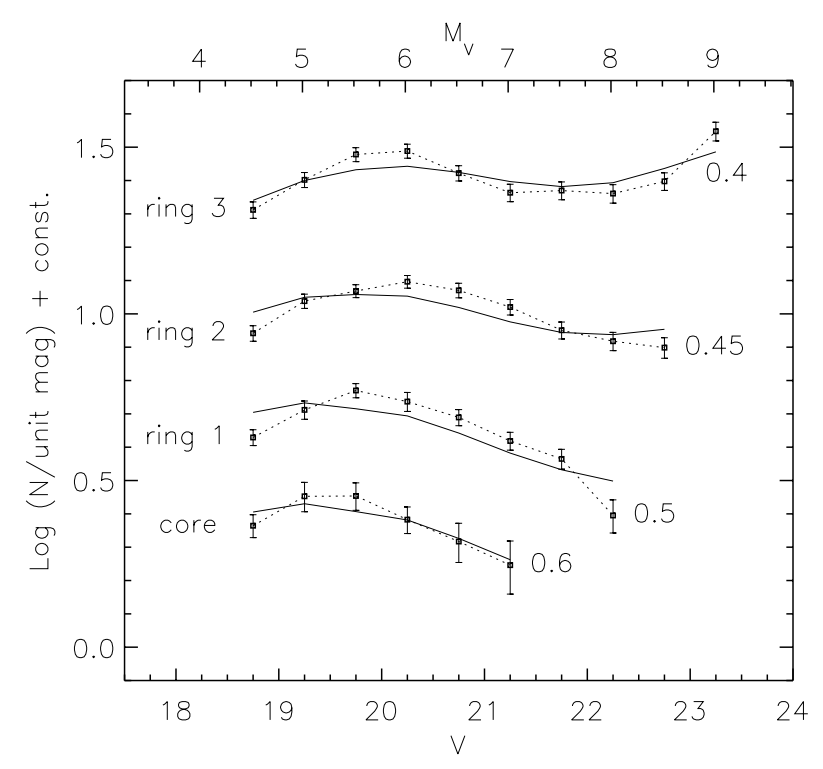

Fig. 5. The squares are the LF of the four regions in NGC 6218, after correction for photometric incompleteness, as given in Table 4. The model LFs that best fit the data are shown as solid lines. The index of their corresponding power-law MF is, from bottom to top, $\alpha=1.4,1.3,0.6$ and 0.1 . A positive index means that the number of stars decreases with mass. The MS stellar mass corresponding to the faintest data point of each LF is indicated, in units of $M_{\odot}$.

observational errors clearly separated from theoretical uncertainties, we prefer to fold a model MF through the derivative of the mass-luminosity $(\mathrm{M}-\mathrm{L})$ relationship and compare the resulting model LF with the observations. The solid lines in Fig. 5 are the theoretical LFs obtained by multiplying a simple power-law MF of the type $\mathrm{d} M / \mathrm{d} m \propto m^{\alpha}$ by the derivative of the $\mathrm{M}-\mathrm{L}$ relationship. Although the MF of GCs is more complex than a simple power-law, particularly at low masses (Paresce \& De Marchi 2000), over the narrow mass range $\left(0.4-0.8 M_{\odot}\right)$ spanned by these observations this simplifying assumption is valid (De Marchi et al. 2005).

With the adopted $\mathrm{M}-\mathrm{L}$ relationship, a distance modulus $(m-M)_{\mathrm{V}}=14.23$ and colour excess $E(B-V)=0.18$ (see Sect. 3), $V=18.8$ corresponds to the TO mass of $0.8 M_{\odot}$. The lowest mass reached in each region is indicated in Fig. 5 and varies from $\sim 0.6 M_{\odot}$ in the core to $\sim 0.4 M_{\odot}$ in ring 3 . The power-law MF indices that best fit the data are indicated in the figure caption and range from $\alpha=1.4$ in the core to $\alpha=0.1$ in the outermost ring near the half-mass radius. The fact that these values are all positive implies that the number of stars is decreasing with mass. With the notation used here, the canonical Salpeter IMF would have $\alpha=-2.35$. For comparison, over the same mass range spanned by the MF of ring 3, the powerlaw index that best fits the MF of NGC 6397 is $\alpha \simeq-1.6$ and that of the other 11 GCs in the sample studied by Paresce \& De Marchi (2000) is of the same order. It is, therefore, clear that NGC 6218 is surprisingly devoid of low-mass stars, at least out to its half-mass radius. 
Table 4. Luminosity functions measured in each of the four regions in which we have divided our NGC 6218 field. For each region, the table gives as a function of the $V$-band magnitude the number of stars per half-magnitude bin before $\left(N_{\mathrm{o}}\right)$ and after $(N)$ completeness correction and the uncertainty on $N$.

\begin{tabular}{ccccccccccccc}
\hline \hline & \multicolumn{3}{c}{ Centre } & \multicolumn{3}{c}{ Ring 1} & \multicolumn{3}{c}{ Ring 2} & \multicolumn{3}{c}{ Ring 3 } \\
$V$ & $N_{\mathrm{o}}$ & $N$ & $\sigma_{\mathrm{N}}$ & $N_{\mathrm{o}}$ & $N$ & $\sigma_{\mathrm{N}}$ & $N_{\mathrm{o}}$ & $N$ & $\sigma_{\mathrm{N}}$ & $N_{\mathrm{o}}$ & $N$ & $\sigma_{\mathrm{N}}$ \\
\hline 18.25 & 182 & 200 & 15 & 335 & 356 & 20 & 345 & 363 & 21 & 228 & 238 & 16 \\
18.75 & 199 & 231 & 18 & 396 & 426 & 23 & 412 & 438 & 24 & 312 & 325 & 18 \\
19.25 & 224 & 284 & 29 & 474 & 515 & 33 & 509 & 547 & 27 & 380 & 400 & 21 \\
19.75 & 216 & 284 & 27 & 530 & 589 & 29 & 534 & 587 & 26 & 448 & 477 & 23 \\
20.25 & 181 & 241 & 22 & 469 & 545 & 36 & 557 & 626 & 27 & 449 & 488 & 24 \\
20.75 & 139 & 207 & 28 & 401 & 489 & 27 & 507 & 590 & 30 & 377 & 419 & 22 \\
21.25 & 104 & 176 & 32 & 320 & 416 & 26 & 436 & 525 & 28 & 322 & 366 & 22 \\
21.75 & 70 & 149 & 26 & 257 & 367 & 25 & 358 & 448 & 26 & 312 & 371 & 23 \\
22.25 & 46 & 135 & 28 & 154 & 248 & 28 & 307 & 415 & 26 & 291 & 364 & 23 \\
22.75 & 21 & 95 & 27 & 166 & 346 & 39 & 250 & 397 & 28 & 285 & 396 & 24 \\
23.25 & 15 & 115 & 46 & 102 & 329 & 46 & 268 & 547 & 47 & 330 & 559 & 36 \\
23.75 & 5 & 83 & 56 & 94 & 522 & 102 & 239 & 724 & 64 & 297 & 707 & 53 \\
24.25 & 2 & 100 & 87 & 45 & 643 & 292 & 177 & 932 & 208 & 190 & 826 & 94 \\
\hline
\end{tabular}

\section{Dynamical structure of NGC 6218}

In order to clarify the origin of this deficiency of low-mass stars, it is useful to compare the radial MF variation implied by Fig. 5 with that expected from the two-body relaxation process that operates in any stellar cluster. To study the dynamical properties of the cluster, we employed the multi-mass Michie-King models originally developed by Meylan $(1987,1988)$ and later suitably modified by Pulone et al. (1999) and De Marchi et al. (2000) for the general case of clusters with a set of radially varying LFs. Our parametric modeling approach assumes energy equipartition among stars of different masses. Each model run is characterised by a MF in the form of a power-law with a variable index $\alpha$, and by four structural parameters describing, respectively, the scale radius $\left(r_{\mathrm{c}}\right)$, the scale velocity $\left(v_{\mathrm{s}}\right)$, the central value of the dimensionless gravitational potential $W_{\mathrm{o}}$, and the anisotropy radius $\left(r_{\mathrm{a}}\right)$.

From the parameter space defined in this way, we selected those models that simultaneously fit both the observed surface brightness profile (SBP) and the central value of the velocity dispersion as measured, respectively, by Trager et al. (1995) and by Pryor et al. (1988). However, while forcing a good fit to these observables constrains the values of $r_{\mathrm{c}}, v_{\mathrm{s}}, W_{\mathrm{o}}$, and $r_{\mathrm{a}}$, the MF can still take on a variety of shapes. To break this degeneracy, we imposed the additional condition that the model LF agrees with that observed at all available locations simultaneously. This, in turn, sets very stringent constraints on the PGMF, i.e. on the MF of the cluster as a whole.

For practical purposes, the model PGMF has been divided into sixteen different mass classes, covering main sequence stars, white dwarfs and heavy remnants, precisely as described in Pulone et al. (1999). We ran a large number of trials looking for a suitable shape of the PGMF such that the local MFs implied by mass segregation would locally fit the observations. As explained in the previous section, in order to keep observational

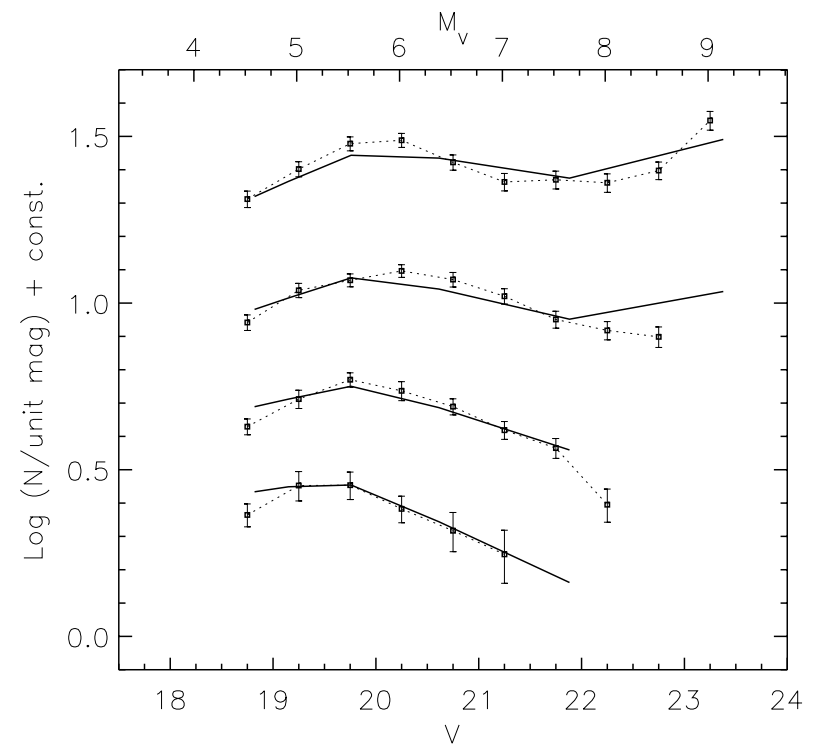

Fig. 6. Same LFs as those of Fig. 5, but here the solid lines show the LFs predicted by our multi-mass Michie-King model at various radii inside the cluster, starting from the same PGMF with index $\alpha=0.1$. The expected radial variation of the LF is fully consistent with the observations.

errors and theoretical uncertainties separate, we converted the model MFs into to LFs, using the same M-L relation, distance modulus and colour excess of Fig. 5, and compared those to the observations.

The set of model LFs that best fits all available observations is shown in Fig. 6 and is drawn from the same PGMF with in$\operatorname{dex} \alpha=0.1$ for stars less massive than $0.8 M_{\odot}$. Figure 7 illustrates the remarkably good fit to the SBP, whereas the values of the best fitting structural parameters are given in Table 5, where they can be compared with those in the literature. The agreement is excellent, apart from a small difference in the value of 


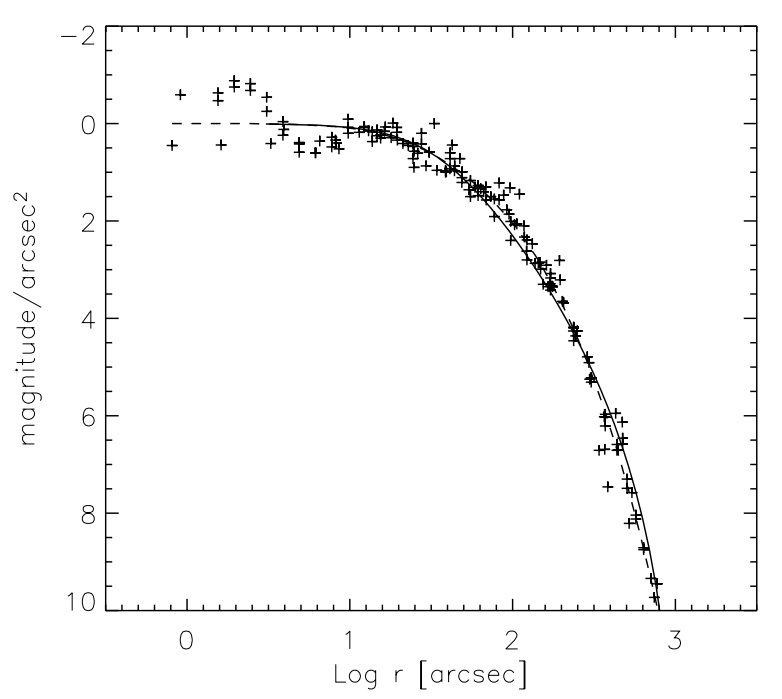

Fig. 7. The surface brightness profile of NGC 6218 (crosses, from Trager et al. 1995) is well reproduced by our dynamical model (solid line). The observations are normalised to the central value of the best fitting profile (dashed line) as given by Trager et al. (1995).

the tidal radius which is, admittedly, not seriously constrained by our data. We note here that we can directly compare the observed SBP with our model since the solid line in Fig. 7 corresponds to stars of $\sim 0.8 M_{\odot}$, which are those contributing most of the cluster's light. As one should expect, stars in different mass classes have different projected radial distributions, with the relative density at any location governed by the relaxation process.

As for the shape of the PGMF, $\alpha=0.1$ implies that it is rather flat or even decreasing with mass. This may have already been inferred by the flat shape of the MF measured near the half-mass radius $r_{\mathrm{h}}$, as it is known that the latter is a good approximation to the cluster's PGMF (Richer et al. 1991; De Marchi et al. 1995). Nevertheless, it is comforting to see that a PGMF of this type is consistent with the shape of the MF observed elsewhere in the cluster, suggesting that the radial change of the MF with radius is the result of the relaxation process and that, therefore, the cluster is in dynamical equilibrium, at least out to the half-mass radius.

This also allows us to set some constraints on the shape of the MF for stars above the TO mass, namely those that have now evolved into degenerate objects (white dwarfs, neutron stars or even black holes). Although not as tightly constrained as for MS stars, the power-law index $\alpha$ of stars more massive than the TO is much closer to the Salpeter value, with $\alpha=-2$ giving the best fit. This parameter determines the fraction of heavy remnants in the cluster, which we find to be of order $60 \%$, and affects the overall distribution of the stars in all other classes of mass, to which the SBP is rather sensitive. We find that values of $\alpha$ larger or smaller than -2 give a progressively worse fit to the SBP, which becomes unacceptable for $\alpha>-1.5$ or $\alpha<-2.5$.

The central velocity dispersion predicted by our model (see Table 5) agrees well with the observed value reported by Pryor $\&$ Meylan (1993) and implies a total cluster mass of $\sim 1.2 \times$ $10^{5} M_{\odot}$, also in line with that of $\sim 1.3 \times 10^{5} M_{\odot}$ derived by those
Table 5. Cluster structural parameters for NGC 6218.

\begin{tabular}{lccc}
\hline \hline Parameter & $\begin{array}{c}\text { Fitted } \\
\text { value }\end{array}$ & $\begin{array}{c}\text { Literature } \\
\text { value }\end{array}$ & Ref. \\
\hline Core radius $r_{\mathrm{c}}$ & $0{ }^{\prime} 6$ & 0.7 & $a$ \\
Tidal radius $r_{\mathrm{t}}$ & $17^{\prime} \cdot 6$ & 20.4 & $a$ \\
Half-mass radius $r_{\mathrm{h}}$ & $3 \prime^{\prime} \cdot 0$ & 2.2 & $a$ \\
Central vel. disp. $\sigma_{\mathrm{v}}$ & $4.5 \mathrm{~km} \mathrm{~s}^{-1}$ & $4.0 \mathrm{~km} \mathrm{~s}^{-1}$ & $b$ \\
\hline
\end{tabular}

$a$ : Harris (1996).

$b$ : Pryor \& Meylan (1993).

authors. The mass to light $(M / L)$ ratio that we obtain depends on the assumed total luminosity of the cluster, which appears to be rather uncertain in the literature. The total $V$-band apparent magnitude varies from $V=6.05$ of Peterson \& Reed (1987; also adopted by Djorgovski 1993) to $V=6.77$ of Webbink (1985). In his recent catalogue, Harris (1996; revision 2003) gives $V=6.70$, corresponding to a total absolute magnitude $M_{V}=-7.31$ and a total luminosity of $\sim 7.2 \times 10^{4} L_{\odot}$. With the latter value, we obtain $M / L_{V}=1.7$ for the whole cluster and $M / L_{V}=1.6$ inside the core radius.

\section{Discussion and conclusions}

What does a flat PGMF tell us about NGC 6218? Using a sample of 12 halo GCs for which deep HST observations are available, Paresce \& De Marchi (2000) showed that their PGMF must directly reflect the properties of the IMF. This conclusion is based on the observation that those clusters have very different properties (total mass, metallicity, concentration and space motion parameters) but they show, in practice, the same or very similar PGMF. This would be hard to justify if the clusters were initially born with very different IMFs. The underlying common IMF, exemplified by that of NGC 6397, is best represented by a power-law distribution that tapers off below $\sim 0.3 M_{\odot}$ (see Paresce \& De Marchi 2000; and De Marchi et al. 2005, for details).

The PGMF of NGC 6218 clearly does not match that of the 12 objects in the sample of Paresce \& De Marchi (2000), because it is remarkably flat. However, at least two other cases of flat or even dropping PGMF have been reported, namely those of NGC 6712 (De Marchi et al. 1999) and Pal 5 (Koch et al. 2004), with NGC 6712 revealing an even stronger deficit of low-mass stars than NGC 6218. On the other hand, there are good observational reasons to believe that both NGC 6712 and Pal 5 have suffered severe tidal disruption that has considerably altered their original distribution of stellar masses. For instance, Pal 5 has well defined tidal tails extending over $10^{\circ}$ across the sky (Odenkirchen et al. 2001). But, more generally, the present total mass and space motion parameters of these clusters imply that they have some of the highest destruction rates in the whole Galactic GC system. Gnedin \& Ostriker (1997) predict a remaining lifetime as low as $\sim 0.3 \mathrm{Gyr}$ for NGC 6712 and $\sim 1 \mathrm{Gyr}$ for Pal 5, whereas Dinescu et al. (1999) give a time to disruption of $\sim 3.9$ Gyr for NGC 6712 and just $\sim 0.1$ Gyr for Pal 5, respectively. Although not in agreement with one another, these 
sets of values are far lower than the average time to disruption, which for both authors is of order $12 \mathrm{Gyr}$ (and thus comparable with the typical GC age).

Quite surprisingly, however, the total mass and space motion parameters of NGC 6218 do not seem to put this cluster in any imminent danger. In fact, the estimated time to disruption for this object varies from $\sim 23.5 \mathrm{Gyr}$ (Gnedin \& Ostriker 1997) to 33 Gyr (Dinescu et al. 1999). Similarly, collisional N-body simulations of a cluster with the properties of NGC 6218 moving in an external tidal field produce a total lifetime for this object of $\sim 29 \mathrm{Gyr}$ (H. Baumgardt, private comm.; see Baumgardt \& Makino 2003 for model details). Assuming a GC age of $~ 12.5 \mathrm{Gyr}$ (Krauss \& Chaboyer 2003), this implies $T_{\mathrm{d}} \simeq 16.5 \mathrm{Gyr}$ for NGC 6218. In other words, none of these models implies a dynamically troubled past for NGC 6218, leaving in principle open the possibility that it was born with a rather flat IMF, at least in the mass range covered by our observations. While such a hypothesis cannot a priori be excluded, this would be the first case known.

On the other hand, models of the dynamical interaction of GCs with the Galactic tidal field are, unfortunately, still subject to large uncertainties. As both Gnedin \& Ostriker (1997) and Dinescu et al. (1999) point out, different assumptions on the initial conditions (cluster orbits) and on the Galactic potential can result in rather different destruction rates for the same cluster. While these models are useful to address the replenishment of the halo over time from the disruption of individual clusters, and therefore helpful to understand the global properties of the GC system and its evolution, they may be still too crude to precisely describe the past history of individual clusters.

We, however, believe that the apparent discrepancy between the predicted value of $T_{\mathrm{d}}$ and the shape of the PGMF of NGC 6218 is simply due to the wrong assumption as to the cluster orbit. In fact, the space motion parameters used by the authors above for NGC 6218 are not consistent with the latest determination of its absolute proper motion based on the Hipparcos reference system. The proper motion measured by Brosche et al. (1991), combined with the radial velocity measurements of Pryor \& Meylan (1993), allowed Dauphole et al. (1996) to put some constraints on the space motion parameters of this cluster. Their findings were confirmed by an independent analysis of the orbit, based on improved absolute proper motions (Scholz et al. 1996), which indicated that NGC 6218 should have a short orbital period $(0.17 \mathrm{Gyr})$ but also that it never ventures closer than $\sim 3 \mathrm{kpc}$ from the Galactic centre, with less than $15 \%$ of its orbit lying within $1 \mathrm{kpc}$ of the Galactic plane. However, a more recent study of the orbit of NGC 6218, based on the new Hipparcos reference system (ESA 1997), has led Odenkirchen et al. (1997) to the conclusion that NGC 6218 has a highly irregular motion. In particular, they find that the low value of the cluster's axial angular momentum forces it to pass the Galactic centre at short distance $\left(R_{\mathrm{p}}=0.6 \mathrm{kpc}\right)$ and to get into strong interaction with the Galactic bulge, since the bulge destruction rate scales with the fourth power of $R_{\mathrm{p}}$ (Dinescu et al. 1999).

With an orbit of this type, the total lifetime of NGC 6218 predicted by the models of Baumgardt \& Makino (2003; H. Baumgardt priv. comm.) would decrease from $29 \mathrm{Gyr}$ to $17 \mathrm{Gyr}$, thus implying a time until disruption of only $T_{\mathrm{d}} \simeq 4.5 \mathrm{Gyr}$, assuming a typical GC age of $\sim 12.5 \mathrm{Gyr}$ as above (Krauss \& Chaboyer 2003). Although not as small as that of NGC 6712 or Pal 5, this value of $T_{\mathrm{d}}$ places NGC 6218 among the clusters at higher risk of disruption and suggests that a considerable fraction of its original stellar population should have been stripped from the cluster.

We can estimate the amount of mass lost by NGC 6218 in the hypothesis mentioned above that all GCs were born with a very similar IMF (Paresce \& De Marchi 2000). As for the latter, we use the tapered power-law proposed by De Marchi et al. (2005) and find that the present total mass due to MS stars is about one fifth of the original $\sim 6 \times 10^{5} M_{\odot}$. This value is in full agreement with the revised calculations of $\mathrm{H}$. Baumgardt (priv. comm.) suggesting an initial mass of $7.6 \times 10^{5} M_{\odot}$. A natural question to pose is when this major mass loss process took place.

If the cluster is in thermo-dynamical equilibrium, one only needs to look at the half-mass relaxation time in order to answer this question. The data are compatible with this hypothesis, since our model imposes the condition of energy equipartition and Fig. 6 and Table 5 show that this is consistent with the radial variation of the MF and the cluster's structural parameters ${ }^{1}$. Under this assumption, the half-mass relaxation time suggested by our models is $t_{\mathrm{rh}} \simeq 0.5 \mathrm{Gyr}$. This value is slightly lower than the $t_{\mathrm{rh}} \simeq 0.7 \mathrm{Gyr}$ given by Djorgovski (1993) and $t_{\mathrm{rh}} \simeq 1$ Gyr of Gnedin \& Ostriker (1997), although still compatible with them, because the MF assumed by these authors is very steep at the low mass end, contrary to what we find, thus resulting in a much larger number of objects in the cluster. In any case, it seems possible to exclude that a major mass loss episode happened in the course of the past $1 \mathrm{Gyr}$ or so, since in that case the cluster should have not yet reached a condition of equilibrium. The fact that tidal tails are very tenuous around NGC 6218 (if at all present; see Lehmann \& Scholz 1997) gives support to this scenario. Mass loss must, therefore, have happened either long ago or continuously, over the cluster lifetime, at a low rate of about $5 \times 10^{3} M_{\odot}$ per orbit.

The present data do not allow us to explore the past dynamical history of NGC 6218 in more detail. Nevertheless, our results show the importance of feeding models of GC distruption with reliable cluster orbits, since the strength and extent of the interaction between the Galaxy and GCs can vary dramatically with the orbit. However, it also depends on the gravitational potential of the Galaxy, and particularly on that of the bulge, thereby making it crucial for models of this type to rely on a solid observational description of the structure of the Galaxy and of its components (thin/thick disc, bulge, halo), which is presently lacking. This information should become available in the near future with the advent of missions such as SIM and

1 We are grateful to an anonymous Referee for pointing out that, although plausible, thermo-dynamical equilibrium is not necessarily implied by the data. In fact, the good fit shown in Fig. 6 and Table 5 is a necessary condition for energy equipartition, but it is not a sufficient one. In principle, there could be models that fit the data equally well but in which the equipartition of energy is not complete. Unfortunately, the method that we followed (Meylan 1987, 1988) does not allow us to investigate this hypothesis. 
Gaia. But already now, if the cluster orbit is reasonably well understood, observations of the cluster PGMF can set meaningful constraints on the mass distribution in the Galaxy. Indeed, the PGMF is a more reliable indicator of the past dynamical history of a cluster than, for instance, its present location and space motion parameters or even the presence and extent of its tidal tails. The position and velocity of a cluster are instantaneous quantities and can vary largely in time. Tidal tails are made up of unbound stars and, as such, are short lived and can only probe the immediate past of a cluster. The PGMF, on the other hand, reflects the integrated effect of the interaction with the Galaxy throughout the whole life of the cluster and provides an indication of the amount of mass lost to the Galaxy. By measuring the PGMF of a sizeable number of clusters, including those with chaotic or anyhow irregular orbits, it should be possible to set meaningful constraints on the form of the Galactic potential well before the availability of astrometric data from interferometric space observatories.

Acknowledgements. It is a pleasure to thank Dana Dinescu and Holger Baumgardt for very useful discussions and the latter also for providing us with the results of his simulations ahead of publication.

\section{References}

Aguilar, L., Hut, P., \& Ostriker, J. 1988, ApJ, 335, 720

Andreuzzi, G., De Marchi, G., Ferraro, F. R., et al. 2001, A\&A, 372, 851

Albrow, M., De Marchi, G., \& Sahu, K. 2002, ApJ, 579, 660

Baraffe, I., Chabrier, G., Allard, F., \& Hauschildt, P. 1997, A\&A, 327, 1054

Baraffe, I., Chabrier, G., Allard, F., \& Hauschildt, P. 1998, A\&A, 337, 403

Baumgardt, H., \& Makino, J. 2003, MNRAS, 341, 247

Baumgardt, H., \& Kroupa, P. 2005, in The three-dimensional universe with Gaia, ESA SP-576, ed. K. O'Flaherty, C. Touron, \& M. Perryman (Noordwijk: ESA), 681

Brosche, P., Geffert, M., Doerenkamp, P., et al. 1991, AJ, 102, 2022

Chabrier, G. 2001, ApJ, 554, 1274

Dauphole, B., Geffret, M., Colin, J., et al. 1996, A\&A, 313, 119

De Marchi, G., \& Paresce, F. 1995, A\&A, 304, 202

De Marchi, G., \& Paresce, F. 1996, ApJ, 467, 658

De Marchi, G., Leibundgut, B., Paresce, F., \& Pulone, L. 1999, A\&A, 343, L9

De Marchi, G., Paresce, F., \& Pulone, L. 2000, ApJ, 530, 342

De Marchi, G., Paresce, F., \& Portegies Zwart, S. 2005, in The initial mass function 50 years later, ASSL 327, ed. E. Corbelli, F. Palla, $\&$ H. Zinnecker (Dordrecht: Springer), 77
Dinescu, D., Girard, T., \& van Altena, W. 1999, AJ, 117, 1792

Djorgovski, S. 1993, in Structure and Dynamics of Globular Clusters, ASP Conf. Ser. 50, ed. S. Djorgovski, \& G. Meylan (San Francisco: ASP), 373

ESA 1997, The Hipparcos and Tycho Catalogues, ESA SP 1200, ed. M. Perryman (Noordwijk: ESA)

Gnedin, O., \& Ostriker, J. 1997, ApJ, 474, 223

Hargis, J., \& Sandquist, E. L. 2004, AJ, 608, 243

Harris, W. 1996, AJ, 112, 1487 (revision 2003)

King, I., Sosin, C., \& Cool, A. 1995, ApJ, 452, L33

King, I., Anderson, J., Cool, A., \& Piotto, G. 1998, ApJ, 492, L37

Koch, A., Grebel, E., Odenkirchen, M., Martinez-Delgado, D., \& Caldwell, J. 2004, AJ, 128, 2274

Krauss, L., \& Chaboyer, B. 2003, Science, 299, 65

Lehmann, I., \& Scholz, R. 1997, A\&A, 320, 776

Leon, S., Meylan, G., \& Combes, F. 2000, A\&A, 359, 907

Meylan, G. 1987, A\&A 184, 144

Meylan, G. 1988, A\&A 191, 215

Meylan, G., \& Heggie, D. 1997, A\&ARv, 8, 1

Odenkirchen, M., Brosche, P., Geffert, M., \& Tucholke, H. 1997, New Astron., 2, 477

Odenkirchen, M., Grebel, E., Rockosi, C., et al. 2001, ApJ, 548, L165

Odenkirchen, M., Grebel, E., Dehnen, W., et al. 2003, AJ, 126, 2385

Paresce, F., \& De Marchi, G. 2000, ApJ, 534, 870

Paresce, F., De Marchi, G., \& Jedrzejewski, R. 1995, ApJ, 442, L57

Pasquali, A., De Marchi, G., Pulone, L., \& Brigas, M. 2004, A\&A, 428, 469

Peterson, C., \& Reed, B. 1987, PASP, 99, 20

Pryor, C., \& Meylan, G. 1993, in Structure and Dynamics of Globular Clusters, ASP Conf. Ser. 50, ed. S. Djorgovski, \& G. Meylan (San Francisco: ASP), 357

Pryor, C., McClure, R., Fletcher, J., \& Hesser, J. 1988, IAU Symp., 126,661

Pulone, L., De Marchi, G., Paresce, F., \& Allard, F. 1998, ApJ, 492, L41

Pulone, L., De Marchi, G., \& Paresce, F. 1999, A\&A, 342, 440

Pulone, L., De Marchi, G., Paresce, F., \& Covino, S. 2003, A\&A, 399, 121

Ratnatunga, K. U., \& Bahcall, J. N. 1985, ApJS, 59, 63

Richer, H., Fahlman, G., Buonanno, R., et al. 1991, 381, 147

Sabbi, E., Ferraro, F., et al. 2006, in preparation

Sato, T., Richer, H., \& Fahlman 1989, AJ, 98, 1335

Scholz, R., Odenkirchen, M., Hirte, S., et al. 1996, MNRAS, 278, 251

Stetson, P. 1987, PASP, 99, 191

Stetson, P. 1994, PASP, 106, 250

Trager, S., King, I., \& Djorgovski, S. 1995, AJ, 109, 1912

Vesperini, E., \& Heggie, D. 1997, MNRAS, 289, 898

Webbink, F. 1985, in Dynamics of star clusters, IAU Symp., 113 (Dordrecht: Reidel), 541 